\title{
Desenvolvimento e Implementação de Indicadores de Colaboração e Participação no Moodle
}

\section{Development and implementation of indications of collaboration and participation in Moodle}

\begin{abstract}
Resumo: A aprendizagem mediada por computador está se expandindo cada vez mais no ensino superior de graduação e pós-graduação, sendo o Moodle um dos ambientes virtuais de aprendizagens mais utilizados. Uma das dificuldades no acompanhamento desses cursos é a visualização global das ações dos usuários (estudantes, tutores, etc.). Objetivou-se nesse trabalho identificar os dados de uso das ferramentas (tabelas de $\log$ ) e construir, a partir desses, indicadores de participação e colaboração global, apresentados em gráficos por período. Focou-se o uso da ferramenta por tutores, professores e coordenadores de cursos a distância (EAD), sendo que essa apresentou potencial uso para moderação das interações e acompanhamento global das atividades.

Palavras-chave: Ambiente virtual de aprendizagem. Avaliação. Indicadores de colaboração e participação.
\end{abstract}

Abstract: The computer mediated learning are increasingly expanding in higher education of graduation and post graduation, being the Moodle one of the most used Virtual Learning Environments. One of the difficulties in monitoring those courses is to achieve a global view for users actions (students, tutor, teacher etc). It aimed in this work, to identify data of Moodle tools (Log tables) and build, from that, global participation and collaboration indicators, presented in graphics by period. It focused on the tool the use by tutors, teachers and coordinators of distance learning (D-Learning), since it has presented a potential use in moderating of the interactions and monitoring of the global activities.

Keywords: Virtual learning environment. Assessment. Indicators of collaboration and participation.

FERRAZ, Paulo Fernando Ozório; OLIVEIRA, Pedro Torres de; HORNINK, Gabriel Gerber. Desenvolvimento e Implementação de Indicadores de Colaboração e Participação no Moodle. Informática na Educação: teoria e prática, Porto Alegre, v. 18, n. 1, p. 85-95, jan./jun. 2015.

\author{
Paulo Fernando Ozório Ferraz
}

Pedro Torres de Oliveira

Gabriel Gerber Hornink

Universidade Federal de Alfenas

\section{Introdução}

0 advento da informática, mais especificamente da internet, vem provocando mudanças significativas nas relações humanas, assim como no modo de conceber, buscar e compartilhar informações (dentro de um gigantesco banco de dados, de acesso instantâneo) e, consequentemente, no modo de pensar, refletindo significativamente nas relações sociais, caracterizando a Era Digital (CASTELLS, 2003, HARVEY, 2001).

Este impacto tecnológico afetou o acesso à informação e, consequentemente, a forma de aprendizagem do homem. A evolução da humanidade é dependente da maneira com a 
qual se concebe e se lê o mundo vivido (MESSA, 2010). Comunicação, tecnologia e educação se constituem num tripé fundamental para a formação do homem do século XXI (FERREIRA; SOARES; LIMA, 2012).

Essas transformações também ocorrem dentro do universo educacional, gerando pressões para que o modo de aprender e ensinar se renovem, saiam do princípio do estudante passivo para o ativo e interativo, incluindo a interatividade digital.

Uma mudança significativa pode ser observada na modalidade de ensino a distância (EAD) desenvolvida no Brasil, desde antes de 1900, no modo clássico, onde o foco estava na informação e de modo unidirecional da informação e ação, como, por exemplo, nos cursos profissionalizantes por correspondência (OLIVEIRA; GASPARIN, 2009).

Diante das transformações sociais e tecnológicas, a concepção do EAD foi se transformando em algo que, atualmente, difere substancialmente do que fora realizado anteriormente, de tal forma que há autores, como Hornink (2010) que denominam essa como ensino online, em que o foco do processo de ensino-aprendizagem reside na aprendizagem colaborativa e na construção de comunidades de aprendizagem online.

Com os avanços nas linguagens e metodologias de programação, pôde-se avançar na construção de ambientes virtuais de aprendizagem (AVA), com inserção de instrumentos digitais para possibilitar os processos de ensino-aprendizagem mediados por computador, na internet (online) (PENTERICH, 2005). Ambientes Virtuais de Aprendizagem, como - Moodle ${ }^{1}$, destacam-se como importantes ferramentas para os cursos EAD. O ambiente

\footnotetext{
${ }_{1}^{1}$ Moodle (Modular Object-Oriented Dynamic Learning Environment)
}

Moodle permite a criação de cursos e inserção de conteúdos, tais como, videoaulas, materiais didáticos, elaboração de lista de exercícios, dentre outros, além de ferramentas para interação (como fórum, wiki, bate-papo).

Destaca-se que o Moodle se constitui de uma plataforma livre, ou seja, encontra-se sob os temos de licença de um software livre, o que possibilitou a constituição de uma grande comunidade (Comunidade Moo$\left.d / e^{2}\right)$ de construção colaborativa do Moodle e seus complementos (Blocos, Módulos, Temas, etc.). Sendo um software livre, possibilitam-se adaptações do mesmo para distintas realidades, assim como o desenvolvimento dos complementos por grupos diversos e seu compartilhamento.

Sobre os métodos de ensino usando AVAs, pode-se questionar sobre as interações em um curso EAD (estudantes, professor, tutor, etc.). Como o professor garante a aprendizagem do estudante? Como saber se o estudante de fato utiliza a plataforma? Como o professor, tutor ou um gestor pode mensurar a participação do estudante no curso ou mesmo ter indícios de sua participação geral/global.

Há uma necessidade importantíssima, do ponto de vista pedagógico, de se ter um acompanhamento que incentiva um relacionamento entre o estudante, o tutor, o professor, dentro do ambiente virtual. Pode-se dizer que é possível ter alguns indícios sobre as ações dos estudantes no Moodle, uma vez que há a coleta de todos dados de ações e interações dos usuários e esses, se bem interpretados, fornecem informações que, relacionadas, compõem um feedback valioso que pode atuar como indicador do processo de aprendizagem.

${ }^{2}$ Comunidade Moodle na Internet: http://www.moodle.org 
Esse acompanhamento pelos formadores (tutores e professores), no sentido de mediar os processos de ensino-aprendizagem, deve considerar a chamada Zona de Desenvolvimento Proximal (ZDP) do estudante, a qual compreende a zona entre o que o estudante é capaz de resolver sozinho com aquela que ele pode desenvolver se auxiliado por alguém mais capaz (VYGOTSKY, 1998), desenvolvendo-se nos estudantes novos habilidades e expandindo a ZDP para novas áreas. Dessa forma, fazem-se necessárias informações subjetivas e objetivas dos estudantes para se compreender tal zona.

Durante o processo de ensino-aprendizagem, conjuntamente com a mediação, entende-se que deva ocorrer a avaliação, de modo formativo e contínuo visando a formação do estudante (SALES; BARROSO; SOARES, 2011), dessa forma, mesclam-se os processos avaliativos com os mediacionais e, para tanto, fazem-se necessário dados das interações nas ferramentas nos ambientes virtuais.

De toda forma, são tantos dados, que é extremamente complexo formar uma visão global sobre o mesmo, assim, faz-se necessário que esse acompanhamento seja sintético, visual e cronológico, ou seja, demanda-se um processo de mineração desses dados (HAN; KAMBER; PEI, 2011).

A proposta de um feedback visual remete ao conceito de cartografar, como sendo o conjunto de estudos e operações científicas, técnicas e artísticas que, tendo por base os resultados de observações diretas ou da análise de documentação, se voltam para a elaboração de mapas, cartas e outras formas de expressão ou representação de objetos, elementos, fenômenos e ambientes físicos e socioeconômicos, bem como a sua utilização (ACI, 1966 apud IBGE, 1999).
Um feedback cartográfico (como um indicador) é composto de valores de variáveis para que seja conceituado e contextualizado. Indicadores são informações que expressam um momento da realidade e permitindo estimar e monitorar por meio de índices de avaliação.

\section{Objetivo/Justificativa}

Objetivou-se a construção de índices (participação e colaboração) que possibilitassem mensurar o acompanhamento pedagógico dos participantes nas disciplinas do AVA Moodle, a partir das ações dos mesmos, em ferramentas disponíveis no ambiente virtual, assim como, a construção de um novo bloco para visualização gráfica desses índices (indeXMoodle ${ }^{3}$ ).

O indeXMoodle propicia ao usuário a visualização sintética e gráfica dos índices a partir de uma abordagem cartográfica, permitindo assim uma melhor análise dos resultados obtidos e, dessa forma, avaliar o desempenho participativo do estudante nas disciplinas em função do tempo.

O indeXMoodle também possibilita a ponderação de relevância de cada ferramenta envolvida (que serão citadas ao decorrer deste artigo) de modo a flexibilizar o cálculo dos índices, de acordo com a realidade pedagógica de cada disciplina e o objetivo da análise.

Do ponto de vista pedagógico, faz-se necessário que cursos EAD, parcialmente ou totalmente lecionados por meio de um AVA, ofereçam formas eficazes de analisar e avaliar as ações e atitudes das partes envolvidas no processo de aprendizagem em uma disciplina, promovendo maior inclusão e interação entre

${ }^{3}$ IndeXMoodle: http://desenvolvimentovirtual.com/hornink/? page_id $=96$ 
as mesmas. As ações e as atitudes cooperativas podem ser identificadas nos ambientes virtuais. A cooperação pressupõe objetivos e valores comuns, trabalho coletivo, respeito mútuo, tolerância, interdependência, negociação constante, entre PENoutros, e.g. (PINTO; BOTELHO, 2012).

Atualmente, pode-se dizer que a maior parte dos AVA's não possui uma ferramenta que demonstre, de forma eficaz, o desenvolvimento do estudante em uma disciplina. Tampouco que possibilite ao professor ou tutor mensurar ações colaborativas e participativas do estudante nas diversas ferramentas que a plataforma oferece, tanto sob uma perspectiva individual, quanto uma perspectiva global.

A partir das discussões anteriores, objetivou-se preencher parte da lacuna existente no processo de aprendizagem EAD, propondo a criação e desenvolvimento de índices de colaboração e participação do usuário na plataforma Moodle.

\section{Método}

O trabalho constou de três etapas gerais (preparatória, embasadora, produção/avaliação), durante as quais o bloco indeXMoodle fora desenvolvido.

A etapa preparatória consistiu na compreensão da estrutura do banco de dados do Moodle e dos blocos, assim como na identificação dos dados existentes nas tabelas.

Para construção deste software, entende-se por participação toda ação mediada pela tecnologia que não envolva diretamente um diálogo ou construção coletiva, com participação ativa. Por exemplo, no Moodle, acesso para visualização ao fórum, visualização do chat, acesso às atividades, acesso ao ambiente.
Também definiu-se colaboração como toda ação mediada pela tecnologia que envolva diretamente um diálogo ou construção coletiva, com participação ativa. Por exemplo, novo tópico ou resposta no fórum, mensagem no chat, texto no wiki.

Durante a etapa embasadora, selecionaram-se os dados relacionados com a participação (ações do usuário de acessos/visualizações) e colaboração (ações de interação) e construíram-se os índices globais de participação e colaboração. Na etapa de produção/ avaliação, desenvolveu-se e testou-se o bloco indeXMoodle.

Durante o desenvolvimento do trabalho se destacaram algumas diretrizes que embasaram todo o desenvolvimento, sendo estas apresentadas a seguir.

\subsection{Desenvolvimento do indeXMoodle}

A construção do bloco se deu baseada na documentação ${ }^{4}$ sobre a construção de Blocos na Comunidade Moodle.

No indeXMoodle, utilizaram-se para o desenvolvimento do front-end, back-end e para a comunicação com o banco de dados do Moodle a linguagem de marcação HTML (HyperText Markup Language), a linguagem de estilo CSS (Cascading Style Sheets), a linguagem de programação PHP (acrônimo recursivo para Hypertext Preprocessor) e a linguagem SQL (Structured Query Language) por meio do DBMS (Data Base Management System) MYSQL.

Para aprimorar a usabilidade, dinamismo e interatividade do sistema foram utilizadas as bibliotecas JavaScript JQuery ${ }^{5}$ 1.8.1 e JavaS-

\footnotetext{
4 Documentação para construção de blocos: https://docs moodle.org/dev/Blocks

${ }^{5}$ jQuery - site oficial: http://jquery.com
} 
cript JQueryUI 1.10 .3 , bibliotecas as quais possibilita interações com elementos HTML, manipulação de eventos, animações, efeitos e que disponibilizam widgets customizáveis. Para a geração e manipulação dos gráficos se utilizou a biblioteca de gráficos HighCharts $3.0^{7}$, a qual é toda baseada em JavaScript e HTML 5.

A estrutura do projeto baseia-se numa forma simples do design pattern MVC (Model-view-controller) que tem como foco o desacoplamento entre as camadas de controle, modelo e visão, o que diretamente favorece a organização do código fonte, aumenta a reusabilidade e a manutenibilidade.

Visto a necessidade de dinamismo nas ações disponíveis da ferramenta e para evitar o recarregamento das páginas, foi aplicada a metodologia AJAX (Asynchronous Javascript and $X M L$ ) por meio do formato JSON (JavaScript Object Notation $)^{8}$, possibilitando assim a realização de requisições assíncronas ao servidor.

\subsection{Modos Gráficos/Visuais de Acom- panhamento}

A proposta do indeXMoodle é centrada na perspectiva gráfico/visual voltada para a necessidade de se cartografar. Dessa forma, toda a perspectiva arquitetural do módulo foi desenvolvida orientada a uma metodologia visual, sintética e objetiva.

\subsection{Coleta de Dados}

A coleta dos dados necessários para o desenvolvimento dos índices de colaboração

\footnotetext{
${ }^{6}$ JQueryUI - site oficial: http://jqueryui.com

${ }^{7}$ HighCharts - site oficial: http://www.highcharts.com

8 JSON - site oficial: http://www.json.org
}

e participação se realizou a partir da tabela mdl_log, tabela na qual são armazenados todos os tipos de ações possíveis no AVA Moodle.

Dessa forma, a partir dos atributos module e action, desta tabela, foi possível a realização da filtragem dos dados de acordo com o módulo, disciplina e participante foco da análise.

Nas Tabelas 1 e 2 são apresentados quais os valores utilizados nos atributos module e action para a filtragem dos dados utilizados no cálculo dos índices.

TABELA 1 - Valores e Atributos Utilizados Para o Índice de Colaboração.

\begin{tabular}{|c|c|c|}
\hline Módulo & Module & Action(s) \\
\hline Fórum & forum & $\begin{array}{c}\text { add post; add } \\
\text { discussion }\end{array}$ \\
\hline Wiki & wiki & add page, edit \\
\hline Chat & chat & talk \\
\hline Glossário & glossary & add entry \\
\hline
\end{tabular}

FONTE: Banco de dados do Moodle.

TABELA 2 - Valores e Atributos Utilizados Para o Índice de Participação.

\begin{tabular}{|c|c|c|}
\hline Módulo & Module & Action(s) \\
\hline Fórum & forum & $\begin{array}{c}\text { view forum; view } \\
\text { discussion }\end{array}$ \\
\hline Wiki & Wiki & view \\
\hline Chat & Chat & view \\
\hline Glossário & glossary & view \\
\hline Questionário & Quis & $\begin{array}{c}\text { attempt; continue } \\
\text { attempt }\end{array}$ \\
\hline Tarefa & assign & submit \\
\hline
\end{tabular}

FONTE: Banco de dados do Moodle.

\subsection{Integração com o Banco de Dados}

Realizou-se a integração com o banco de dados utilizando-se o DBMS MySQL, por meio do arquivo de configuração config.php, localizado no diretório raiz do Moodle. Por meio deste arquivo, torna-se possível a captura dos 
dados de acesso ao banco, tais quais configurados durante sua instalação.

$\mathrm{O}$ arquivo config.php prepara um vetor de variáveis com valores referentes acerca das configurações básicas do Moodle, como nome, usuário e senha de acesso ao banco.

Para acessar este vetor foi criado um script que executa a importação do arquivo config. php e realiza a manipulação dos dados de acesso ao banco em um arquivo nomeado config.txt, de configuração do próprio indeXMoodle. Este script será chamado toda vez que o indeXMoodle for acessado. Caso seja o primeiro acesso, o arquivo é criado, caso contrário, utiliza-se o já existente.

Uma vez armazenadas as variáveis de acesso ao banco de dados do AVA Moodle, o módulo indeXMoodle passa a ter livre acesso às tabelas do banco para efetuar as consultas necessárias para o cálculo dos índices.

\subsection{Método de Desenvolvimento dos Índices}

Para o desenvolvimento dos índices se necessitou definir quais módulos do Moodle seriam analisados, quais ações do módulo seriam consideradas como colaboração, quais ações seriam consideradas como participação e, por último, uma forma de alcançar valores representativos por meio de equações matemáticas preestabelecidas.

Visto que um índice global único (tanto de colaboração, quanto de participação) não propiciaria uma análise congruente do desenvolvimento pedagógico do estudante na disciplina, desenvolveram-se índices globais de colaboração e participação em intervalos semanais.

Os índices são representados em um gráfico, cujo eixo $Y$ representa a pontuação (de 0 a 10, onde 10 é referente ao participante mais colaborativo ou participativo) e o eixo $X$ as semanas a partir do momento da criação do vínculo participante/disciplina até o momento atual. Dessa forma, o desenvolvimento é apresentado por meio da pontuação em função do tempo.

Porém, somente os índices globais não são suficientes para uma análise da atuação do estudante. Faz-se interessante uma representação de índices individuais das ferramentas analisadas, ou seja, índices que representem a colaboração e participação em cada módulo do Moodle analisado. Desta forma, realiza-se um comparativo entre a utilização dos diferentes módulos e da utilização de todos de um modo geral.

Definiram-se os seguintes índices:

- Índice de Colaboração Global: calculado a partir dos índices de colaboração individual dos módulos definidos;

- Índice de Colaboração Individual: calculado a partir das colaborações do módulo em questão, podendo ser este módulo o fórum, wiki, chat ou glossário;

- Índice de Participação Global: calculado a partir dos índices de participação individual dos módulos definidos;

- Índice de Participação Individual: calculado a partir das participações do módulo em questão, podendo ser este módulo o fórum, wiki, chat, glossário, questionário ou tarefa.

Como apresentado nas Tabelas 1 e 2, a base de informações para o cálculo dos índices foram os módulos e ações a seguir:

- Fórum: consideradas como colaboração as ações de iniciar uma nova discussão 
ou responder a uma existente e como participação a leitura do fórum;

- Wiki: consideradas como colaboração as ações de adicionar uma nova página ou editar uma já existente e como participação a leitura do wiki;

- Chat: considerada como colaboração a ação de conversar por meio do mesmo e como participação a leitura do chat;

- Glossário: considerada como colaboração a ação de adicionar um novo termo e como participação a leitura do glossário;

- Questionário: consideradas como participação as ações de finalizar um questionário ou dar continuidade em um questionário em aberto;

- Tarefa: considerada como participação a ação de submeter o envio de um arquivo.

\section{Resultados e Discussões}

Obteve-se a produção do bloco indexMoodle, nos padrões descritos na comunidade Moodle, sendo que a instalação do mesmo ocorre por meio do plugin de instalação, no modo administrador do ambiente.

Como resultado, obtiveram-se as equações matemáticas de cálculo dos índices e a construção da ferramenta indeXMoodle para manipulação e visualização dos índices.

Os resultados serão explanados nas subseções a seguir.

\section{1 Índices}

Definiram-se os cálculos dos índices globais (participação e colaboração) como uma média ponderada, ou seja, são obtidos por meio da soma entre a multiplicação dos índices individuais pelo fator peso correspondente, dividida pela soma total dos pesos (Equação 1, 2).

$$
i C=\frac{(p e F * i C F)+(p e W * i C W)+(p e C * i C C)+(p e G * i C G)}{t P e}
$$

Equação 1 - Índice de colaboração global (iC).

$i P=\frac{(p e F * i P F)+(p e W * i P W)+(p e C * i P C)+(p e G * i P G)+(p e Q * i P Q)+(p e T * i P T}{t P e}$

Equação 2 - Índice de participação global (iP).

Desenvolveram-se os cálculos dos índices individuais (índices correspondentes cada módulo do Moodle) em três partes. Primeiramente é realizado o somatório de todas colaborações ou participações realizadas na disciplina, a partir da data de criação do vínculo participante/disciplina até a data atual em questão. Em seguida é calculada a média simples de colaboração ou participação, dividindo o somatório obtido anteriormente pelo número atual de semanas. Por fim, o índice individual é alcançado por meio da multiplicação do total de colaborações ou participações do participante que está sendo analisado, dividido pela média que foi obtida (Equações 3, 4).

Para atingir um resultado mais representativo, todos os índices gerados são normalizados numa escala de 0 a 10, onde 10 representa o maior índice semanal existente.

$i C I=\frac{t C P * t C C}{m C} \quad m C=\frac{t C A}{k+1} \quad t C A=\sum_{k=0}^{n} t C C$

Equação 3 - Índice de colaboração individual (iCI).

$i P I=\frac{t P P * t P C}{m P} \quad m P=\frac{t P A}{k+1} \quad t C A=\sum_{k=0}^{n} t P C$

Equação 4 - Índice de participação individual (iPI). 
iC - Índice de Colaboração Global; iCF - Índice de Colaboração do Fórum; iCW - Índice de Colaboração do Wiki; icC - Índice de Colaboração do Chat; iCG - Índice de Colaboração do Glossário.

iP - Índice de Participação Global; iPF - Índice de Participação do Fórum; iPW - Índice de Participação do Wiki; iPC - Índice de Participação do Chat; iPG - Índice de Participação do Glossário; iPQ - Índice de Participação do Questionário; iPT - Índice de Participação da Tarefa.

iCI - Índice de Colaboração Individual; tCP - Total de colaborações do participante; tCC - Total de colaborações no curso; mC - Média de colaborações; tCA - Total acumulado de colaborações.

iPI - Índice de Participação Individual; tPP - Total de participações do participante; tPC Total de participações no curso; mPP - Média de participações; tPA - Total acumulado de participações.

tPe - Total soma dos pesos; peF - Fator peso Fórum; peW - Fator peso Wiki; peC - Fator peso Chat; peG - Fator peso Glossário; peQ - Fator peso Questionário; peT - Fator peso Tarefa; n - Número de semanas.

\subsection{0 indeXMoodle}

Desenvolveu-se o sistema de modo que possibilitasse um fácil e rápido acesso pelo usuário, com todas opções da ferramenta em uma única tela (Figura 1), baseando-se nos princípios de usabilidade na Web (NIELSEN; LORANGER, 2007), ou seja, um sistema intuitivo, com alta navegabilidade, necessidade de poucos cliques, visualização em poucas telas, etc. Para geração dos gráficos, basta selecionar um participante do grupo e as distribuições dos pesos por ferramenta de acordo com o objetivo da análise.

Visto a quantidade de participantes que podem estar vinculados a uma disciplina, o indeXMoodle oferece dois filtros para a seleção de participantes. O filtro papel apresenta todos os papéis cadastrados no banco de dados do Moodle, separando os ativos dos inativos, e o filtro de grupos apresenta todos os grupos existentes no curso selecionado.

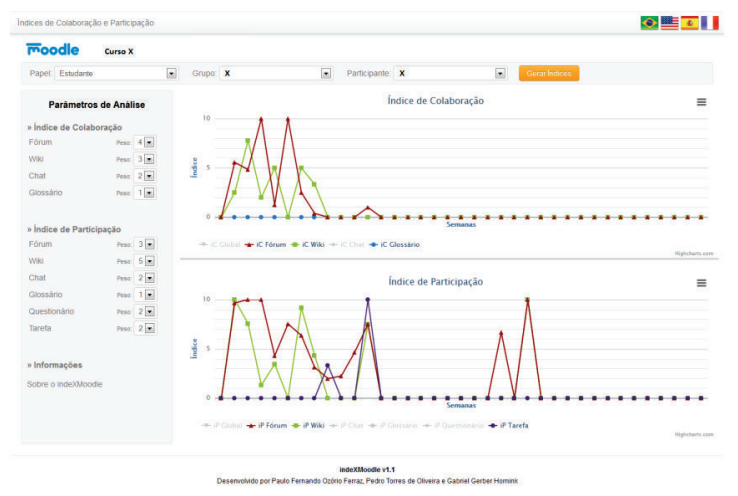

FIGURA 1 - Visão geral do indeXMoodle FONTE: TORRES et al., 2014.

O AVA Moodle possui uma grande comunidade envolvida e ativa, sendo amplamente conhecido e utilizado em diversos países. Dessa forma, construiu-se o indeXMoodle com a opção de ser utilizados em quatro línguas distintas, sendo elas: Português Brasileiro (padrão), Inglês, Espanhol e Francês; possibilitando também facilmente a inserção de novas linguagens por meio, basicamente, dos arquivos de tradução.

O filtro papel apresenta todos os papéis ativos e não ativos (porém, os inativos não podem ser selecionados) cadastrados no banco de dados do Moodle relacionados com a disciplina, ou seja, é possível gerar índices para todos aqueles que participam do curso, inerente ao papel do participante, permitindo a geração de índices também para gestor, professor, tutor, coordenador, etc.

O filtro de grupos apresenta todos os grupos existentes na disciplina, desta maneira a 
ferramenta pode gerar os índices de um participante em relação a um ou todos os grupos.

Por meio de requisições $A J A X$, os índices são calculados e os gráficos são renderizados no centro da página. Os índices são calculados por períodos semanais, tornando visível a progressão das atividades do participante avaliado.

Nos gráficos, o eixo $Y$ representa o índice, variando entre 0 e 10 , enquanto o eixo $X$ representa o período semanal correspondente. Para a visualização de um índice no ponto ( $x$, y) é preciso posicionar o ponteiro do mouse sob o mesmo, para que a caixa de informação apareça, conforme a Figura 1.

Oferece-se também no indeXMoodle a possibilidade de customização dos pesos de participação de cada ferramenta para composição dos índices (Figura 2), aumentando a flexibilidade da análise ponderada nos cálculos dos índices (cada usuário pode dar maior ou menor importância para cada ferramenta, de acordo com a proposta didático-pedagógica de cada curso.

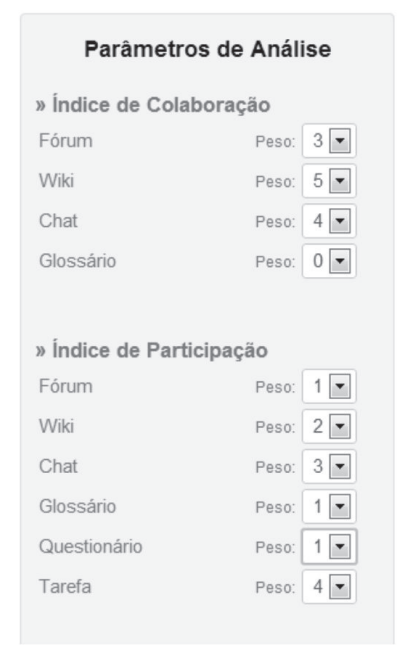

FIGURA 2 - Detalhe das Opções Para Mudança nos Pesos de Cada Ferramenta. FONTE: TORRES et al., 2014.

\section{Conclusão}

O ser humano possui em sua natureza uma capacidade inata de interpretação de um conjunto de dados quando apresentados em um contexto visual. Segundo Bosi (1993), os psicólogos afirmam que o homem moderno recebe as informações por meio de imagens: "[...] O homem de hoje é um ser predominantemente visual. [...]" (BOSI, 1993, p. 67).

Quanto maior a quantidade de dados a serem analisados, mais difícil se torna a extração do conhecimento contido neles. Dessa forma, buscam-se novas maneiras de como representar um conjunto de dados de acordo com o domínio de sua necessidade, principalmente com o uso de representações visuais sintéticas, que partem de um modelo cartográfico de representar as ações do homem.

Visto a inexistência de ferramentas semeIhantes, o desenvolvimento do indeXMoodle se baseou na premissa do desenvolvimento dos indicadores (participação e colaboração) e de um meio de visualização gráfica direta, usual, flexível e que proporcionasse formas concretas de análise do desenvolvimento pedagógico dos participantes das disciplinas, resultando em uma forma mais clara e sintética dos dados, possibilitando a interpretação do conjunto de dados coletados.

Uma vez que cada disciplina apresenta suas necessidades e particularidades distintas, ou seja, diferentes importâncias para cada ferramenta do Moodle (chat, fórum, wiki, etc.), fez-se necessário oferecer flexibilização nos índices durante o uso da ferramenta, a partir da possibilidade de escolha de pesos diferentes para cada ferramenta, o que resultou em grande flexibilidade e adaptação da ferramenta para cada realidade, desempenhando um papel importante no desenvolvimento dos 
indicadores para acompanhamento da disciplina.

Espera-se que, com o aumento da comunidade que faz utilização de AVAs e, principalmente com o aumento do oferecimento de cursos EAD por diversas instituições pelo mundo, mais e mais ferramentas como o indeXMoodle sejam desenvolvidas para o auxílio no processo de ensino-aprendizagem por meio de plataformas virtuais e possibilitando novos meios para construção dos conhecimentos.

\section{Referências}

BOSI, A. Fenomenologia do Olhar. In: NOVAES, A. (Org.). O Olhar. São Paulo: Cia. das Letras, 1990. p. 65-87.

CASTELLS, M. The internet Galaxy: Refletions on the Internet, Business and society. Oxford: Oxford University Press, 2003.

FERREIRA, E.B.; SOARES, A.B.; LIMA, C. Aprimoramento Conceitual e Uso de Demonstrações Matemáticas: um estudo de caso sobre a Geometria Dinâmica e as pesquisas de campo com ambientes computacionais de ensino. Revista Brasileira de Informática na Educação, v. 30, n. 3, p. 13-25, 2012.

HAN, J.; KAMBER, M.; PEI, J. Data Mining: Concepts and Techniques. 3. ed. Burlington: Elsivier, 2011. HARVEY, D. Condição Pós-Moderna. 16. ed. São Paulo: Loyola, 2001.

HORNINK, G.G. Cartografando Online: caminhos da informática na escola com professores que elaboram conhecimentos em formação contínua. 2010. Tese (Doutorado) - Curso de Pós-graduação em Ensino e História de Ciências da Terra, Universidade Estadual de Campinas, 2010, Campinas, BR-SP.

IBGE. Noções Básicas de Cartografia. Rio de Janeiro: IBGE, 1999.

MESSA, W.C. Utilização de Ambientes Virtuais de Aprendizagem - AVAS: a busca por uma aprendizagem significativa. Revista Brasileira de Aprendizagem Aberta e a Distância, v. 9, p. 1-49, 2012. Disponível em: <http://www.abed.org.br/revistacientifica/Revista_PDF_Doc/2010/2010_2462010174147. pdf> Acesso em: 06 ago. 2014.

NIELSEN, J.; LORANGER, H. Usabilidade na WeB. Rio de Janeiro: Elsevier, 2007.

OLIVEIRA, D.E.M.B.; GASPARIN, J.L. A Expansão da Educação a Distância no Brasil e os Aspectos Pedagógicos Esquecidos. In: SEMINÁRIOS DE PESQUISA DO PORGRAMA DE PÓS-GRADUAÇÃO EM 
EDUCAÇÃO, 2009, Maringá. Anais eletrônicos. Maringá: Universidade Estadual de Maringá, 2009. P. 1-10. Disponível em: <http://www.ppe.uem.br/publicacoes/seminario_ppe_2009_2010/pdf/2009/39. pdf > Acesso em: 06 ago. 2014.

PENTERICH, 2005 COMPLETAR REFERÊNCIA

PINTO, I.M.; BOTELHO, S.C. Ambientes Tecnológicos Lúdicos de Autoria (ATLA): criando espaços de ensino e aprendizagem. Revista Brasileira de Informática na Educação, v. 20, n. 3, p. 81-96, 2012

SALES, G.L.; BARROSO, G.C.; SOARES, J.M. Um Indicador de Aprendizagem Não-Linear Para EaD Online Fundamentado no Modelo de Avaliação Learning Vectors (LV). Revista Iberoamericana de Evaluación Educativa, v. 4, n. 2, p. 155-180, 2011.

TORRES, P.; TORRES, P.; Ferraz, P.F.O. ; Ferraz, P.F.O. ; HORNINK, G.G. indeXMoodle. 2014. Patente: Programa de Computador. Número do registro: BR5120140003566, data de registro: 08/04/2014, título: "indeXMoodle", Instituição de registro:INPI - Instituto Nacional da Propriedade Industrial.

VYGOTSKY, L.S. A Formação Social da Mente. 6. ed. São Paulo: Martins Fontes, 1998.

Submetido para avaliação em 12 de novembro de 2014

Aprovado para publicação em 28 de abril de 2015.

Paulo Fernando Ozório Ferraz - Universidade Federal de Alfenas, Alfenas, BR-MG. E-mail: nandooferraz@gmail.com Pedro Torres de Oliveira - Universidade Federal de Alfenas, Alfenas, BR-MG. E-mail: torres.op@gmail.com Gabriel Gerber Hornink - Universidade Federal de Alfenas, Alfenas, BR-MG. E-mail: gabrielbio@gmail.com 